\title{
An adaptive control scheme for hyperbolic partial differential equation system (drilling system) with unknown coefficient
}

\author{
HAMED SHIRINABADI FARAHANI, HEIDAR ALI TALEBI and MOHAMMAD BAGHERMENHAJ
}

The adaptive boundary stabilization is investigated for a class of systems described by second-order hyperbolic PDEs with unknown coefficient. The proposed control scheme only utilizes measurement on top boundary and assume anti-damping dynamics on the opposite boundary which is the main feature of our work. To cope with the lack of full state measurements, we introduce Riemann variables which allow us reformulate the second-order in time hyperbolic PDE as a system with linear input-delay dynamics. Then, the infinite-dimensional time-delay tools are employed to design the controller. Simulation results which applied on mathematical model of drilling system are given to demonstrate the effectiveness of the proposed control approach.

Key words: drilling systems, adaptive control, hyperbolic partial differential equation, wave equation, boundary control

\section{Introduction}

We investigate boundary stabilization for a class of linear second-order hyperbolic PDE system with uncertainty coefficient on a finite space domain. The general issue addressed in this paper is how to deal with the wave in a one-dimensional form, as considered e.g. when modeling the dynamics of an elastic slope vibrating around its rest position. Particularly, we consider the wave equation describing the dynamics of the deformation denoted by $z(x, t)$.The research activities in boundary control field were devoted to parabolic PDEs in the early 2000s [1]. In recent years, however, more attention has been given to the hyperbolic PDEs and in particular to the stabilization of such dynamics [2-5].Many physical systems can be described by first-order hyperbolic PDEs, such as traffic flow, heat exchangers [20].Subsequently, in [6] systems with unknown input delay, i.e., an important class of infinite dimensional systems with first-order hyperbolic PDE dynamics is tackled. In [18-19] sufficient condition for exponential stability

The Authors are with Amirkabir University of Technology, Tehran, Iran. H. Talebi is the corresponding author, e-mail: alit@aut.ac.ir. Other e-mails: h.shirinabadi@aut.ac.ir and menhaj@aut.ac.ir

Received 5.06.2016. Revised 25.08.2016. 
for various class of nonlinear first-order hyperbolic PDE system is given. In [21], backstepping approaches have been used for first-order hyperbolic PDEs to achieve stability.

To the best of our knowledge, adaptive output-feedback boundary control problem has not yet been developed for the second-order hyperbolic PDEs especially when the dynamics are unstable, namely the wave equation. Wave and beam equations have been addressed in [7-9], however, the dynamics are assumed to be stable.In [10], adaptive boundary control of unstable wave equation is studied by measuring all state $z(x, t) x \in$ $[0,1]$ which is not feasible in real application. Recently in [17], an adaptive control law for wave equation is designed by measuring down and top boundary, simultaneously.

This paper is devoted to the boundary stabilization of uncertain hyperbolic PDE system. Here, we introduce the main ideas for back-stepping control of hyperbolic PDEs, the most basic of which is the wave equation. The main distinguished feature of a wave equation is that it is second order in time.

We consider mathematical model of drilling system [11], as a case study, which described (in linear form) by the second-order in time hyperbolic PDE subject to boundary conditions with unmatched parametric uncertainty.

We use the modified Riemann variables to reformulate the plant model as a linear input-delay model cascaded with a transport equation opposite of the input propagation direction. This structure allows us to reconstruct the delayed bottom velocity from topboundary measurement. The Lyapunov methodology is then used for stability analysis. Both control and parameter estimation approaches utilize top-boundary measurement only, which is the main feature of our work. Toward this objective, first an invertible infinite-dimensional back-stepping transformation is introduced to transform the original system into a target system, from which it is much easier to design the desired controller and implement the performance analysis.Then, for the target system, a dynamic compensation for the unknown parameter is given by an adaptive technique and projection operator. Subsequently, based on this technique and the certainty equivalence principle, an adaptive controller is constructed to stabilize the target system in a certain sense. Finally, by the invertibility of the infinite-dimensional back-stepping transformation, the controller designed for the target system can stabilize the original system in the foregoing sense.

The rest of the paper is organized as follows. In Sec. 2, we introduce the mathematical modeling of drilling system and then present adaptive controller in Sec. 3 followed by the statement of the main stability theorem and its proof. We finally end our paper with a numerical simulations to illustrate the effectiveness of our proposed approach in Sec. 4. Concluding remarks and possible further research lines are presented in Sec. 5.

Notation: \|\|$_{L^{2}}$ denotes the norm in $L^{2}(0,1)$ space, defined by $\|u\|_{L^{2}(0,1)}^{2}=\int_{0}^{1}|u|^{2} d x$ for all functions $u \in L^{2}(0,1)$. Similarly, $H^{2}(0,1)$ is the set of functions $u \in H^{2}(0,1)$ such that $\int_{0}^{1}|u|^{2}+\left|u_{x}\right|^{2}+\mid u_{x x}^{2} d x$ is finite. ( $u_{x}$ stands for the partial derivative of the function $u$ with respect to $x$ ). Also, for $(a, b) \in R^{2}$ such that $a<b$, we define the standard projection operator on the interval $[a, b]$ as a function of two scalar arguments $f$ (denoting the 
parameter being updated) and $g$ (denoting the nominal update law) as

$$
\operatorname{Proj}_{[a, b]}(f, g)=g\left\{\begin{array}{lll}
0 & \text { if } & f=a \text { and } g<0 \\
0 & \text { if } \quad f=b \text { and } g>0 \\
1 & \text { otherwise. }
\end{array}\right.
$$

\section{Mathematical modeling}

To evaluate the performance of the proposed adaptive control approach, we consider a drilling system model [11], shown in Fig. 1. The main process of oil well drilling, which is depicted in Fig.1, includes in creation of a narrow deep hole in the ground until the oil reservoir is reached [11]. This system consists of a bit, tool for cutting rock, drill pipes, drill collars and rotatory table which provides torque on drill pipe for penetrating into ground.

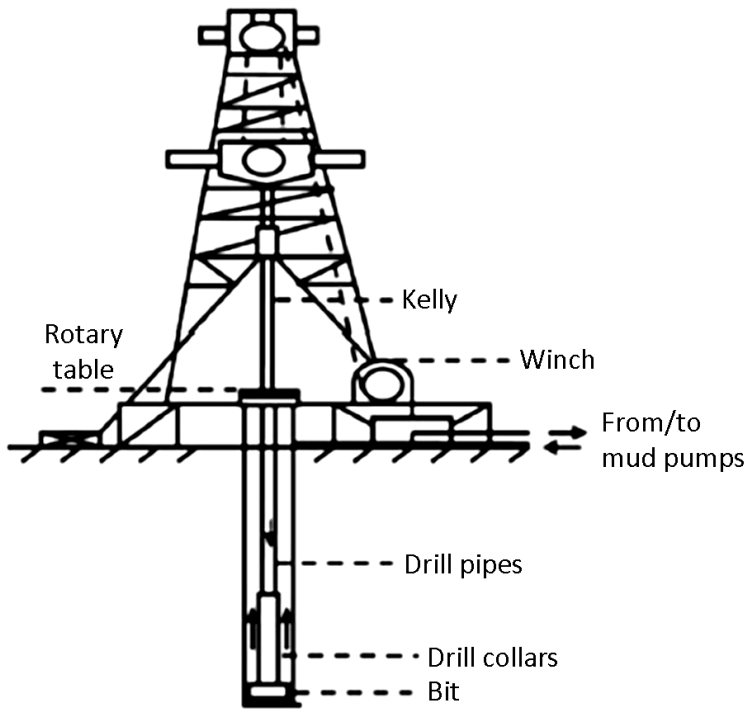

Figure 1: Drilling system

A distributed parameter model of drilling system is described by the second-order hyperbolic PDEs as follow [11]:

$$
G J \frac{\partial^{2} \theta(\xi, t)}{\partial \xi^{2}}-I \frac{\partial^{2} \theta(\xi, t)}{\partial t^{2}}-\beta \frac{\partial \theta(\xi, t)}{\partial t}=0, \quad \xi \in[0, L], t \geqslant 0
$$


with boundary conditions

$$
\begin{gathered}
I_{B} \frac{\partial^{2} \theta}{\partial t^{2}}(0, t)=G J \frac{\partial \theta}{\partial \xi}(0, t)+T\left(\frac{\partial \theta}{\partial t}(0, t)\right) \\
G J \frac{\partial \theta}{\partial \xi}(L, t)=\Omega(t)
\end{gathered}
$$

where $\theta(\xi, t):[0, L] \times R^{+} \rightarrow R$ (angle of rotation) and $\frac{\partial \theta}{\partial t}(\xi, t)$ (angular velocity) are the system states with $\left(\theta(\cdot, 0), \frac{\partial \theta}{\partial t}(\cdot, 0)\right) \in H_{1}([0, L]) \times L_{2}([0, L]), \frac{\partial \theta}{\partial t}$ and $\frac{\partial \theta}{\partial \xi}$ denote the partial derivatives of $\theta(\xi, t)$ with respect to $t$ and $\xi$, respectively, $T\left(\frac{\partial \theta}{\partial t}(0, t)\right)$ is the torque which is a nonlinear function of the bit speed which is uncertain, and $\Omega(t)$ : $R^{+} \rightarrow R$ is the control law coming from the rotor, $I$ is the inertia, $G$ is the shear modulus and $J$ is the geometrical moment of inertia.

In the sequel, the damping coefficient $\beta$ is assumed to be zero and without loss of generality, $L$ is assumed to be equal one. By choosing $q=\frac{d T}{d \frac{\partial \theta}{\partial t}}\left(\left(\frac{\partial \theta}{\partial t}\right)^{r e f}\right)$ (called anti-damping coefficient) where $\left(\frac{\partial \theta}{\partial t}\right)^{r e f}$ is a given angular velocity to be achieved, the distributed mathematical model reduces to the unidimensional wave equation

$$
\begin{gathered}
\frac{\partial^{2} \theta(\xi, t)}{\partial \xi^{2}}=\rho^{2} \frac{\partial^{2} \theta(\xi, t)}{\partial t^{2}} ; \quad \xi \in[0,1], t \geqslant 0 ; \rho=\sqrt{\frac{I}{G J}} \\
\frac{\partial^{2} \theta}{\partial t^{2}}=c \frac{\partial \theta}{\partial \xi}(0, t)+c q \frac{\partial \theta}{\partial t}(0, t) ; \quad c=\frac{G J}{I_{B}} \\
\frac{\partial \theta}{\partial \xi}(1, t)=g \Omega(t)=U(t) ; \quad g=\frac{1}{G J} .
\end{gathered}
$$

Our objective is to design a feedback law $U(t)$ to ensure dissipativity of the system, despite uncertainty in anti-damping coefficient $q>0$, which does not employ the entire distributed state, but only the top boundary value measurement. We only measure angular velocity of the top boundary i.e. signal $\frac{\theta}{t}(1, \cdot)$, for all time. This assumption arises in a new formulation in which we need only the velocity at the boundary for all time. The main challenge here is instability of dynamics (5) with unmatched parametric uncertainty, since $q$ acts on the lower boundary while the controller is applied on the opposite boundary. To deal with parameter uncertainties, as common in adaptive control approach, we assume that there exist a fixed and known constants $Q_{\min }, Q_{\max }$ such that $Q_{\min }<q<Q_{\max }, \forall x \in[0,1]$.

First, without loss of generality we assume $\rho^{2}=1$ and reformulate the plant (4)-(6) by introducing Riemann variables $u(\xi, t)=\frac{\partial \theta(\xi, t)}{\partial t}-\frac{\partial \theta(\xi, t)}{\partial \xi}$ and $v(\xi, t)=\frac{\partial \theta(\xi, t)}{\partial t}+\frac{\partial \theta(\xi, t)}{\partial \xi}$ as follows: 


$$
\begin{gathered}
\frac{\partial u(\xi, t)}{\partial t}=-\frac{\partial u(\xi, t)}{\partial \xi} \\
\frac{\partial v(\xi, t)}{\partial t}=\frac{\partial v(\xi, t)}{\partial \xi} \\
v(1, t)=\frac{\partial \theta}{\partial t}(1, t)+U(t)=W(t) \\
\frac{\partial^{2} \theta}{\partial t^{2}}(0, t)-c(1-q) \frac{\partial \theta}{\partial t}(0, t)=c v(0, t) \\
u(0, t)=2 \frac{\partial \theta}{\partial t}(0, t)-v(0, t) .
\end{gathered}
$$

ODE equation (10) can be represented in standard state space form as:

$$
\dot{X}(t)=\left[\begin{array}{c}
\dot{x}_{1}(t) \\
\dot{x}_{2}(t)
\end{array}\right]=\left[\begin{array}{cc}
0 & 1 \\
0 & c(q-1)
\end{array}\right]\left[\begin{array}{l}
x_{1}(t) \\
x_{2}(t)
\end{array}\right]+\left[\begin{array}{l}
0 \\
c
\end{array}\right] v(0, t)
$$

where $x_{1}(t)=\theta(0, t)$ and $x_{2}(t)=\frac{\partial \theta}{\partial t}(0, t)$ are the states of linear ODE system (10). It is obvious that this system is unstable for $q>1$. The control objective is to design $v(1, t)$ and in turns $U(t)$ (from (9)) to achieve asymptotic stability of (10).

\section{The proposed adaptive control design}

In this section, similar to [10] we present an infinite dimensional back-stepping transformation, using which system (5) is transformed into a target system. Then, based on this transformation, our proposed adaptive controller is designed, which can stabilize the original system in the desired sense. If $q$ is known, the back-stepping transformation $\left(x_{2}(t), u, v\right) \rightarrow\left(x_{2}(t), u, \omega\right)$ is given as follows:

$$
\omega(\xi, t)=v(\xi, t)-(1-c-q)\left[\int_{0}^{\xi} k(\xi, y) v(y, t) d y+\lambda(\xi) \frac{\partial \theta}{\partial t}(0, t)\right]
$$

where kernel $k(\xi, y)$ and $\lambda(\xi)$ are to be designed later.

Now, by taking the derivative of $\omega$, we obtain:

$$
\begin{aligned}
& \frac{\partial \omega(\xi, t)}{\partial \xi}= \\
& \frac{\partial v(\xi, t)}{\partial \xi}-(1-c-q)\left[k(\xi, \xi) v(\xi, t) d y+\int_{0}^{\xi} \frac{\partial k(\xi, y)}{\partial \xi} v(y, t) d y+\lambda^{\prime}(\xi) \frac{\partial \theta}{\partial t}(0, t)\right]
\end{aligned}
$$




$$
\begin{aligned}
& \frac{\partial \omega(\xi, t)}{\partial t}= \\
& \frac{\partial v(\xi, t)}{\partial t}-(1-c-q)\left[k(\xi, y) \frac{\partial}{\partial t} v(y, t) d y+\lambda(\xi) \frac{\partial^{2} \theta}{\partial t^{2}}(0, t)\right] .
\end{aligned}
$$

Then, using integration by part, and using (10), we can get

$$
\begin{aligned}
& \frac{\partial \omega(\xi, t)}{\partial t}=\frac{\partial v(\xi, t)}{\partial t}-(1-c-q)[k(\xi, \xi) v(\xi, t)-k(\xi, 0) v(0, t)- \\
& \left.-\int_{0}^{\xi} \frac{\partial k(\xi, y)}{\partial y} v(y, t) d y+\lambda(\xi)\left(c(q-1) \frac{\partial \theta}{\partial t}(0, t)+c v(0, t)\right)\right] .
\end{aligned}
$$

In case where $q$ is known, the target system is given by [10]:

$$
\begin{gathered}
\frac{\partial \omega(\xi, t)}{\partial t}=\frac{\partial \omega(\xi, t)}{\partial \xi} \\
\omega(1, t)=0 .
\end{gathered}
$$

Then, by substituting (14) and (16) into (17) and using (18), we can get

$$
\begin{gathered}
\frac{\partial k(\xi, y)}{\partial y}+\frac{\partial k(\xi, y)}{\partial \xi}=0 \\
\lambda^{\prime}(\xi)-c(q-1) \lambda(\xi)=0 \\
k(\xi, 0)=c .
\end{gathered}
$$

The solution of the PDE (19) and ODE (20) can then be given as:

$$
\begin{gathered}
k(\xi, y)=c e^{c(q-1)(\xi-y)} \\
\lambda(\xi)=e^{c(q-1) \xi} .
\end{gathered}
$$

In [10], the ODE system with input delay was modeled as a first order hyperbolic PDE and the back-stepping transformation used to design the controller, however, the adaptive case has not been addressed.

In case where the damping factor $q$ is unknown, the back-stepping transformation (13) can be changed to:

$$
\omega(\xi, t)=v(\xi, t)-(1-c-\hat{q})\left[\int_{0}^{\xi} \hat{k}(\xi, y, t) v(y, t) d y+\hat{\lambda}(\xi, t) x_{2}(t)\right]
$$

where $\hat{q}$ is the estimate of unknown $q$. 
Taking the time derivative of the transformation (24) and using the dynamics (8)-(12) we obtain

$$
\begin{gathered}
\frac{\partial \omega(\xi, t)}{\partial t}=\frac{\partial v(\xi, t)}{\partial t}-\int_{0}^{\xi} \frac{\partial k(\xi, y, t)}{\partial t} v(y, t) d y-\int_{0}^{\xi} k(\xi, y, t) \frac{\partial v(y, t)}{\partial t} d y-\lambda(\xi) \dot{x}_{2}(t)= \\
\frac{\partial v(\xi, t)}{\partial \xi}-\int_{0}^{\xi} \frac{\partial k(\xi, y, t)}{\partial t} v(y, t) d y-\int_{0}^{\xi} k(\xi, y, t) \frac{\partial v(\xi, t)}{\partial \xi} d y-\lambda(\xi)\left(c(q-1) x_{2}(t)+c v(0, t)\right) .
\end{gathered}
$$

Then, using integration by parts and taking the derivative of (24) with respect to $x$ and noting that

$$
\frac{\partial k(\xi, y, t)}{\partial t}+\frac{\partial k(\xi, y, t)}{\partial y}=0
$$

the original system (7)-(12) can be transformed into following target system:

$$
\begin{gathered}
\dot{x}_{2}(t)=-c c_{0} x_{2}(t)+c \omega(0, t)+c \widetilde{q}(t) x_{2}(t) \\
\frac{\partial \omega(\xi, t)}{\partial t}=\frac{\partial \omega(\xi, t)}{\partial \xi}-c \widetilde{q}(t) x_{2}(t) \lambda(\xi)+\dot{\hat{q}}(t) f(\xi, t) \\
\omega(1, t)=0 \\
\frac{\partial u(\xi, t)}{\partial t}=-\frac{\partial u(\xi, t)}{\partial \xi}
\end{gathered}
$$

Where $\widetilde{q}(t)=q-\hat{q}(t)$ and $\hat{q}(t)$ is the estimate of unknown parameter $q$, and $f(\xi, t)$ is defined as:

$$
\begin{aligned}
& f(\xi, t)=\left(\frac{1}{1-c_{0}-\hat{q}(t)}-c \xi\right) \lambda(\xi) x_{2}(t)-c \int_{0}^{\xi} k(\xi, y, t)(\xi-y) v(y, t) d y+ \\
& +\left(\frac{1}{1-c_{0}-\hat{q}(t)}\right) \int_{0}^{\xi} k(\xi, y, t) v(y, t) d y .
\end{aligned}
$$

Now, by plugging the transformation (24) into (27) and using (8-10), after a lengthy but straightforward computation we obtain

$$
\begin{aligned}
\hat{k}(\xi, y, t) & =c e^{c(\hat{q}(t)-1)(\xi-y)} \\
\hat{\lambda}(\xi) & =e^{c(\hat{q}(t)-1) \xi} .
\end{aligned}
$$

Given the back-stepping transformation (24), the following theorem states the main contribution of the paper which summarizes the proposed control law, adaptation rule and stability proof. 
Theorem 7 (Stability of the target system (17) and (18)). Consider the target system (25)-(28). Then, by using control law (38), and adaptation law (36), the zero equilibrium of target system is exponentially stable in the sense of the following system norm:

$$
Y(t)=\left(\left\|\frac{\partial \omega(\cdot, t)}{\partial t}\right\|_{L^{2}(0,1)}\left\|\frac{\partial \omega(\cdot, t)}{\partial \xi}\right\|_{L^{2}(0,1)}+\left|x_{2}(t)\right|^{2}\right)^{\frac{1}{2}} .
$$

Proof Consider the following candidate Lyapunov function

$$
V(t)=E(t)+\frac{\widetilde{q}(t)^{2}}{\gamma}
$$

where

$$
E(t)=\log \left[1+\left(x_{2}(t)\right)^{2}+\int_{0}^{1} e^{\xi} \omega(\xi, t)^{2} d \xi+\int_{0}^{1} e^{1-\xi} u(\xi, t)^{2} d \xi\right] .
$$

In the sequel, we will omit the arguments when the notation is obvious. The time derivative of $V(t)$ is given by:

$$
\dot{V}(t)=\frac{1}{1+\psi(t)}\left[2 x_{2} \frac{\partial x_{2}}{\partial t}+\int_{0}^{1} 2 e^{\xi} \omega \frac{\partial \omega}{\partial t} d \xi+\int_{0}^{1} 2 e^{1-\xi} u \frac{\partial u}{\partial t} d \xi\right]-\frac{2}{\gamma_{q}} \dot{\hat{q}}(t) \widetilde{q}(t)
$$

where

$$
\psi(t)=\left(x_{2}(t)\right)^{2}+\int_{0}^{1} e^{\xi} \omega(\xi, t)^{2} d \xi+\int_{0}^{1} e^{1-\xi} u(\xi, t)^{2} d \xi .
$$

Using Young and Cauchy-Schwartz inequalities yields in:

$$
\exists \widetilde{M}>0 \text { s.t. }\left|2 \dot{\widetilde{q}}(t) \int_{0}^{1} e^{\xi} \omega(\xi, t) f(\xi, t) d \xi\right| \leqslant \gamma_{q} \widetilde{M}\left(x_{2}(t)^{2}+\|\omega(t)\|^{2}\right) .
$$

Then by choosing the following adaptation law

$$
\dot{\hat{q}}(t)=\frac{a \gamma_{q}}{1+\psi(t)} \operatorname{Proj}_{\left[Q_{\min }, Q_{\max }\right]}\{g(t, \hat{q}(t)), \hat{q}(t)\}
$$

where

$$
\left.g(t, \hat{q}(t))=x_{2}(t)\left(x_{2}(t)+\left(\hat{q}(t)+c_{0}-1\right) \in_{(} t-1\right)^{t} f(\tau) e^{(\tau-t+1)(c(\hat{q}(t)-1)+1)} d \tau\right)
$$

and by using property of projection operator together with (26)-(29), equality (34) can be expressed as:

$$
\dot{V}(t) \leqslant-\operatorname{frack} \psi(t)\left(x_{2}(t)^{2}+\|\omega(\xi, t)\|^{2}+\|u(\xi, t)\|^{2}\right) ; \quad k>0 .
$$


Finally, plugging (24) into (28) imposes the following control law:

$$
\begin{aligned}
& v(1, t)=W(t)=(1-c-\hat{q}(t))\left[\int_{0}^{1} \hat{k}(1, y, t) v(y, t) d y+\hat{\lambda}(\xi) x_{2}(t)\right]= \\
& =(1-c-\hat{q}(t))\left[\int_{0}^{1} c e^{c(\hat{q}-1)(1-y)} W(t+y-1) d y+e^{a(\hat{q}-1) \xi_{x}} x_{2}(t)\right] .
\end{aligned}
$$

To finish, by suitable change of variable $t+y-1=\tau$ the control law can be expressed as follows:

$$
\left.W(t)=(1-c-\hat{q}(t))\left[\int_{(} t-1\right)^{t} c e^{c(\hat{q}-1)(1-y)} W(\tau) d \tau+e^{a(\hat{q}-1) \xi} x_{2}(t)\right] .
$$

Now consider the following inverse back-stepping transformation $\left(x_{2}(t), u, \omega\right) \rightarrow$ $\left(x_{2}(t), u, v\right)$ as

$$
v(\xi, t)=\omega(\xi, t)-\left(c_{0}+\hat{q}-1\right) \int_{0}^{\xi} m(\xi, y, t) \omega(y, t) d y-\rho(\xi) x_{2}(t)
$$

where kernel $m(\xi, y, t)$ and $\rho(\xi)$ can be computed by applying Laplace transformation in $\xi$ to both sides of (24) and using (30-31) can be expressed as follows:

$$
\begin{gathered}
m(\xi, y, t)=c e^{c c_{0}(\xi-y)} \\
\rho(\xi)=e^{c c_{0} \xi} .
\end{gathered}
$$

Corollary 1 (Stability of the original system) Consider the plant (4-6), control law (38), and adaptation law (36), here exist $M_{0}, N_{0}>0$ such that $E(t) \leqslant M_{0}\left(e^{N_{0} E(0)}-1\right)$.

Proof We consider the following Lyapunov function candidate

$$
E(t)=(q-\hat{q}(t))^{2}+\int_{0}^{1}\left[\frac{\partial \theta}{\partial \xi}(\xi, t)\right]^{2} d \xi+\int_{0}^{1}\left[\frac{\partial \theta}{\partial t}(\xi, t)\right]^{2} d \xi+x_{2}(t)^{2}
$$

Notice that

$$
\frac{\partial \theta(\xi, t)}{\partial t}=\frac{u(\xi, t)+v(\xi, t)}{2}
$$

and

$$
\frac{\partial \theta(\xi, t)}{\partial \xi}=\frac{v(\xi, t)-u(\xi, t)}{2}
$$


Time differentiation of $E(t)$ yields in

$$
\begin{aligned}
& \dot{E}(t)=-2 \dot{\hat{q}}(t)+\int_{0}^{1}\left(\frac{\partial v}{\partial t}(\xi, t)-\frac{\partial u}{\partial t}(\xi, t)\right)\left(\frac{v(\xi, t)-u(\xi, t)}{2}\right) d \xi+ \\
& +\int_{0}^{1}\left(\frac{\partial v}{\partial t}(\xi, t)+\frac{\partial u}{\partial t}(\xi, t)\right)\left(\frac{v(\xi, t)+u(\xi, t)}{2}\right) d \xi+2 x_{2}(t) \dot{x}_{2}(t) .
\end{aligned}
$$

Then plugging parameter update law (37) and calculate control law noticing that by (9) $U(t)=v(1, t)-\frac{\partial \theta}{\partial t}(1, t)(1, t)$, using (36) and applying Young and Cauchy-Schwartz inequalities, it can be seen that there exist $M_{0}, N_{0}>0$ such that

$$
E(t) \leqslant M_{0}\left(e^{N_{0} E(0)}-1\right) .
$$

\section{Simulation results}

In this section, we present numerical simulation to illustrate the effectiveness of the proposed controller. Similar to [11] we focus on trajectory of the form $\theta^{\text {ref }}(\xi, t)=$ $-T\left(\omega_{r}\right) \xi+\omega_{r} t+u_{0}$ where $\omega_{r} \equiv \frac{\partial \theta^{r e f}(\xi, t)}{\partial t}$ is uniform rotatory speed with the reference control input $\bar{U}=-T\left(\omega_{r}\right)$.

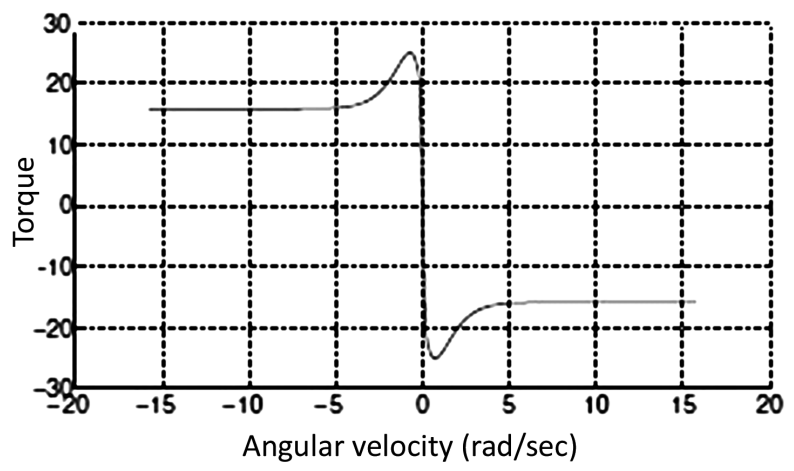

Figure 2: Function presenting rock-on-bit friction

Our objective is to design an adaptive controller that exponential stability of system (4)-(6) be guaranteed.

According to our proposed scheme, we consider only a measurement of top velocity $\omega(t)=\frac{\partial \theta}{\partial t}(1, t)$. The parameters of the model used in simulations are taken from [12] to ease performance comparisons and gathered in Tab. 1 . 
Table 3: List of parameter used in simulations

\begin{tabular}{|c|c|c|}
\hline \hline Symbol & Description & Value \\
\hline \hline$L$ & Length of the drillpipe & $2000 \mathrm{~m}$ \\
\hline$I_{d}$ & Inertia of the drillpipe per unit length & $0.095 \mathrm{~kg}$ \\
\hline$I_{b}$ & Inertia of the BHA & $311 \mathrm{kgm}^{2}$ \\
\hline$G$ & Shear modulus & $79.310^{9} \mathrm{kgm}^{2}$ \\
\hline$J$ & Geometric moment of inertia & $1.1910^{-5} \mathrm{~m}^{4}$ \\
\hline$\beta$ & Drill string damping & 0 \\
\hline$T_{\text {tobdyn }}$ & Torque on the bit parameter & $7500 \mathrm{~N}$ \\
\hline$\alpha_{1}, \alpha_{2}, \alpha_{3}$ & Friction parameters & $5.5,2.2,3500$ \\
\hline$c_{b}$ & Viscous damping torque at the bit & $0.03 \mathrm{Nm} \mathrm{sec} / \mathrm{rad}$ \\
\hline
\end{tabular}

Velocity reference is chosen $\frac{\partial \theta^{\text {ref }}(\xi, t)}{\partial t}=3$. Therefore the unknown parameter $q=$ 0.21 . Initial parameter's estimate is $\hat{q}(0)=0.25$. The parameter estimate evolution is depicted in Fig. 3. By control input as depicted in Fig. 4 where adaptive control is turned on at $t=9 \mathrm{sec}$, stabilization of the drill string using back-stepping controller is achieved and shown in Fig. 5. It means that by control law (39) the stick-slip vibrations of drill string are reduced. Also in Fig. 5, the velocity at surface follows a similar trend delayed by $0.5 \mathrm{sec}$ which corresponds to the time needed for the control law to propagate back to the surface. As shown in Fig. 3, the estimate of $q$ converges but not to the unknown parameter, even if stabilization is satisfied.

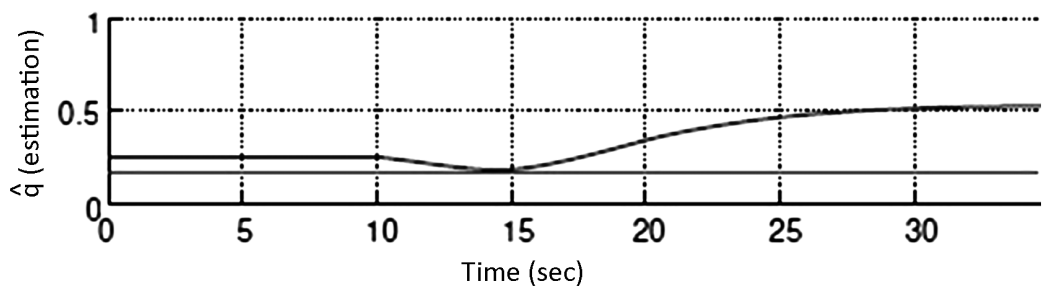

Figure 3: Parameter estimate evolution 


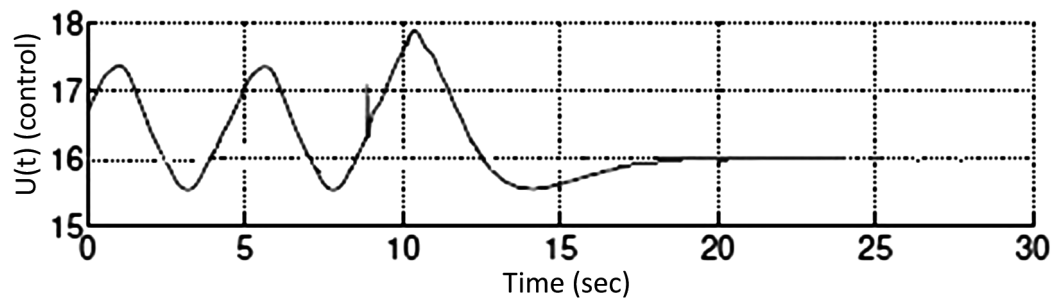

Figure 4: Control signal: input-adaptive controller is turned on after $9 \mathrm{sec}$

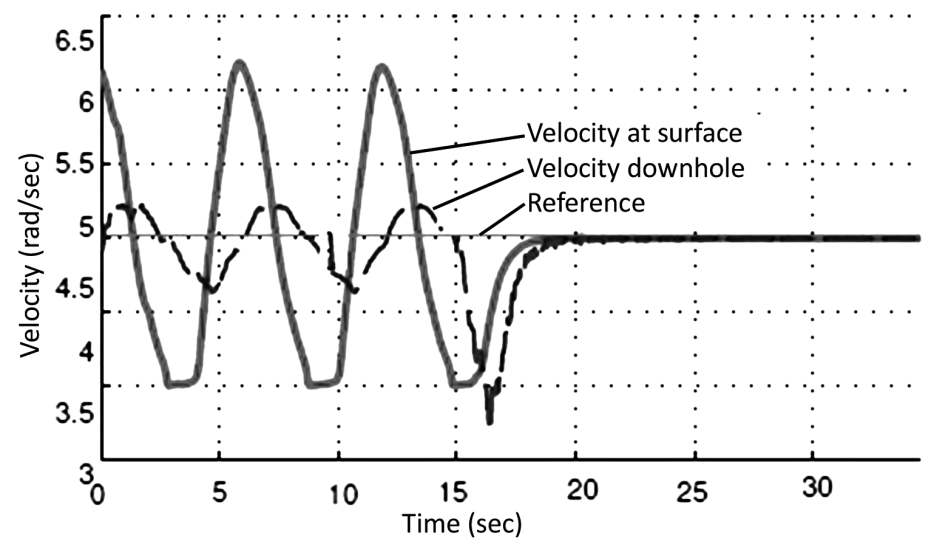

Figure 5: Velocity evolution: adaptive controller turned on after $9 \mathrm{sec}$

\section{Conclusion}

In this paper, we consider a class of second-order hyperbolic partial differential equation (wave equation) with unknown coefficient and propose an adaptive controller. The achievement of this new control method is that it does not require the measurements of the entire system state but only of top boundary values. The extension of this technique to other types of boundary conditions, is a topic of the further work.

\section{References}

[1] A. BAlOGH, W.-J. LiU and M. KRSTIC: Stability enhancement by boundary control in 2D channel flow. IEEE Trans on Automatic Control, 46 (2001), 1696-1711.

[2] J.-M. Coron, G. Bastin and B. D'Andrea Novel: . Dissipative boundary conditions for one-dimensional nonlinear hyperbolic systems. SIAM J. on Control and Optimization, 47 (2008), 1460-1498. 
[3] J.-M. Coron, B. D'Andrea Novel and G. Bastin: A strict Lyapunov function for boundary control of hyperbolic systems of conservation laws. IEEE Trans. on Automatic Control, 52 (2007), 2-11.

[4] J.-M. Coron, R. VAzQuez, M. KRstic and G. BAstin: .Local exponential $\mathrm{H}_{2}$ stabilization of a $2 \times 2$ quasilinear hyperbolic system using backstepping. SIAM J. on Control and Optimization, 51 (2013), 2005-2035.

[5] H. ShIRINABADI and H.A. TALEBI: Lyapunov stability analysis of special class of PDE systemsControl. 2nd Int. Conf. on Instrumentation and Automation, (2011), 648-653.

[6] D. BResch-Pietri and M. KRstiC: Adaptive trajectory tracking despite unknown input delay and plant parameters. Automatica, 45 (2009), 2074 Ü2081.

[7] T. KoBAYASHI: Low-gain adaptive stabilization of infinite-dimensional secondorder systems. J. Mathematical Analysis and Applications, 275 (2002), 835-849.

[8] T. KobAyAshi: Adaptive stabilization of infinite-dimensional semilinear secondorder systems. IMA J. Mathematical Control and Information, 20 (2003), 137-152.

[9] T. KobAYASHI: Stabilization of infinite-dimensional second-order systems by adaptive PI controllers. Mathematical Methods in the Applied Sciences, 24 (2001), 513-527.

[10] M. KRSTIC: Adaptive control of an anti-stable wave PDE. Dynamics of Continuous, Discrete and Impulsive Systems, 17 (2010), 853-882.

[11] B.SAldivar, S.Mondi, J.JACQUES and L.V. RASVAn: Stick-slip oscillations in oill well drilings: Distributed parameter and neutral type retarded model approaches. 18th IFAC World Congress, Italy, (2011).

[12] QI-ZHI ZHANG, YU-YAO HE, LIN LI and NuRZAT: Sliding mode control of rotary drilling system with stick slip oscillation. Int. Workshop on Intelligent Systems and Applications, China, (2010), 1-4.

[13] P.A. IoAnNou and B. FIDAN: Adaptive Control Tutorial. Society for Industrial Mathematics, 2006.

[14] A. Serrarens, M. Van de Molengraft, J. KoK and L. Van den Steen: $H_{\infty}$ control for suppressing stick-slip in oil well drillstrings. IEEE Control Systems, 18(2), (1998), 19-30.

[15] C. Sagert, F. Di Meglio, M. Krstic and P. Rouchon: Backstepping and flatness approaches for stabilization of the stick-slip phenomenon for drilling. IFAC Symp. on System, Structure and Control, 2013. 
[16] M. KRISTIC: Boundary Control of PDEs: A Course on Backstepping Designs. Society for Industrial Mathematics, 2008.

[17] D. PIETRI and M. KRISTIC: Output-feedback adaptive control of wave PDE with boundary anti-damping. Automatica, 50 (2014), 1407-1415.

[18] C. PRIEU and F. MAZENC: ISS-Lyapunov function for time varying hyperbolic systems of balance law. Mathematics of Control, Signals, and Systems, 24 (2012), 111-134.

[19] F. Castillo, E. Witrant, C. Prieure and L. Dugrad: Dynamic boundary stabilization of hyperbolic systems. Proc. 51 st IEEE Conf. on Decision and Control, Maui, USA, (2012).

[20] D. BRESCH-PIETRI and M. KRSTIC: Delay-adaptive predictor feedback for systems with unknown long actuator delay. IEEE Trans. on Automatic Control, 55(9), (2010), 2106-2112.

[21] M. KRStiC and A. Smyshlyaev: Backstepping boundary control for first-order hyperbolic PDEs and application to systems with actuator and sensor delays. Systems and Control Letters, 57(9), (2008), 750-758. 